Supporting Information

\title{
Self-Powered Multifunctional Electronic Skin for Smart Anti-Counterfeiting Signature System
}

Hang Guo, ${ }^{1} \mathrm{Ji}$ Wan, ${ }^{2}$ Hanxiang Wu, ${ }^{2}$ Haobin Wang, ${ }^{2}$ Liming Miao, ${ }^{2}$ Yu Song, ${ }^{2}$ Haotian Chen, ${ }^{1}$ Mengdi Han, ${ }^{3 *}$ and Haixia Zhang ${ }^{1,2 *}$

${ }^{1}$ Academy for Advanced Interdisciplinary Studies, Peking University, Beijing 100871, China.

2 National Key Laboratory of Science and Technology on Micro/Nano Fabrication, Peking University, Beijing 100871, China.

${ }^{3}$ Center for Bio-Integrated Electronics, Northwestern University, Evanston, Illinois 60208, USA

*Co-corresponding-authors: hanmd1990@gmail.com, zhang-alice@pku.edu.cn

\section{Supplementary Figures}

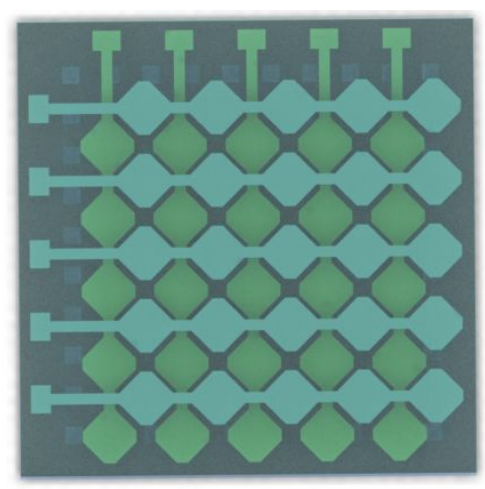

Figure S1. Top view of the TENG section.

TENG Part

PENG Part

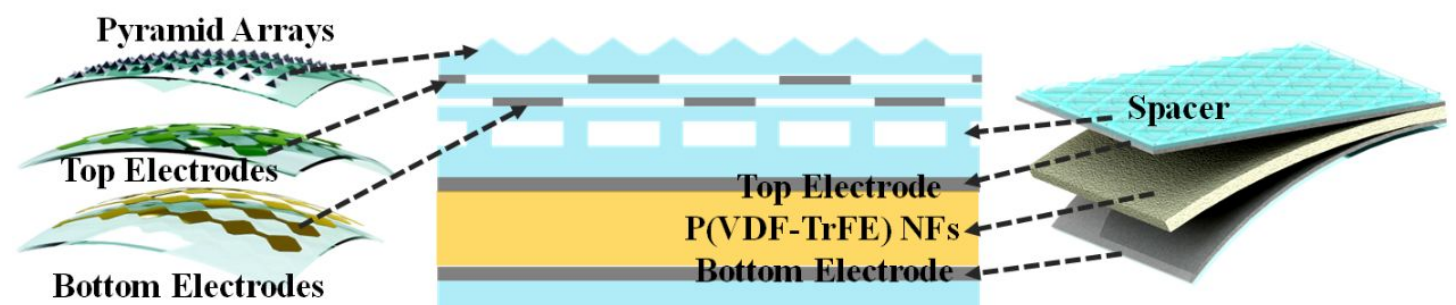

Figure S2. The structure of the whole device by integrating TENG and PENG. 
Figure S3a shows the movement of the object in a single direction along a single electrode (B2) on the multifunctional electronic skin. As can be seen from the respective electrode signal diagrams on the right side, there are five consecutive signal peaks on electrode B2, which correspond exactly to the five repeating patterns designed on electrode B2. For the top electrodes $\mathrm{T} 1$ to $\mathrm{T} 5$, the moving object sequentially passes through the respective electrodes at the top as it slides along the electrode B2, so that the peaks of the signals on the electrodes T1 to T5 appear in sequence, which is completely matched to the actually tested electrical signal. Apart from this, figure S3b-d displays the output signals of each electrode in other three different cases, such as sliding along the diagonal, longitudinal and transverse directions on the surface of the device. With different motion trajectories, the order and period of the output signals will also change. 
(a)

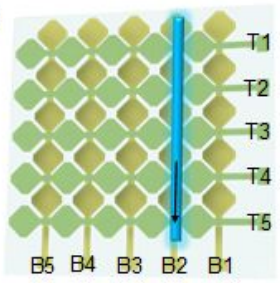

(b)

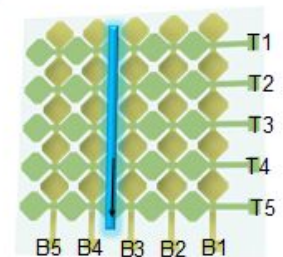

(c)

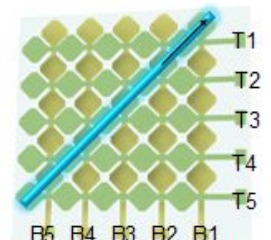

(d)

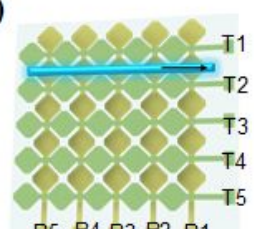

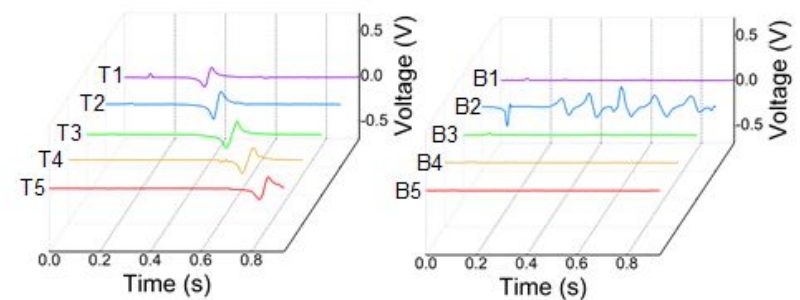
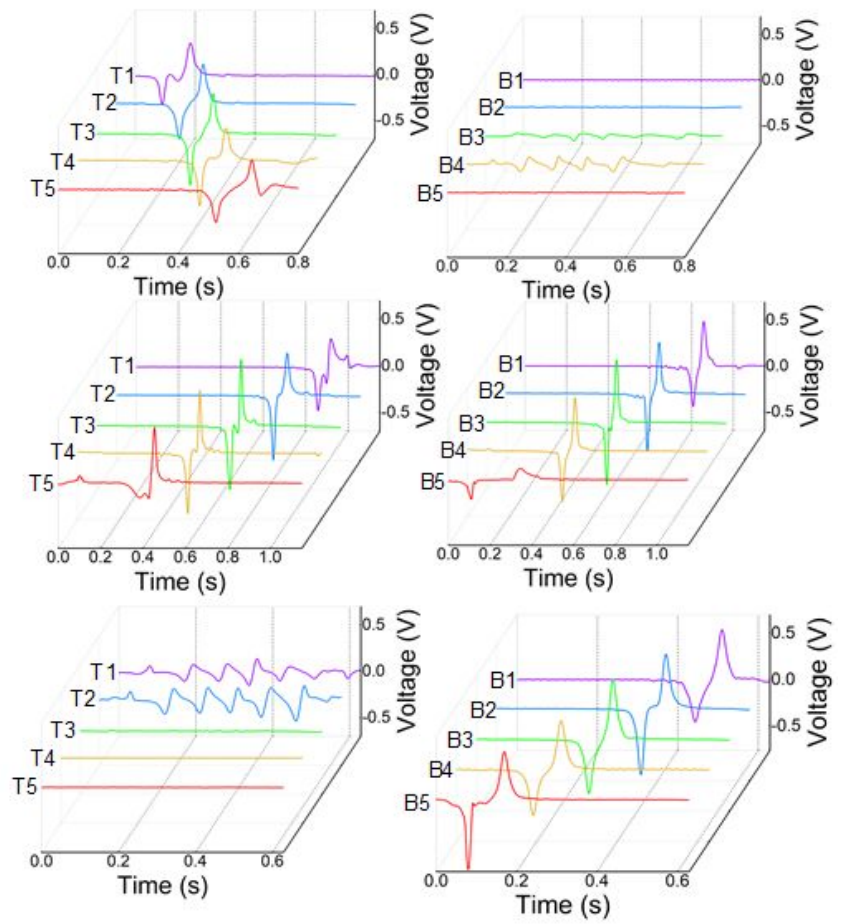

Figure S3. (a-d) The output signals of each electrode when object slides along the single electrode (B2), longitudinal, diagonal and transverse directions.

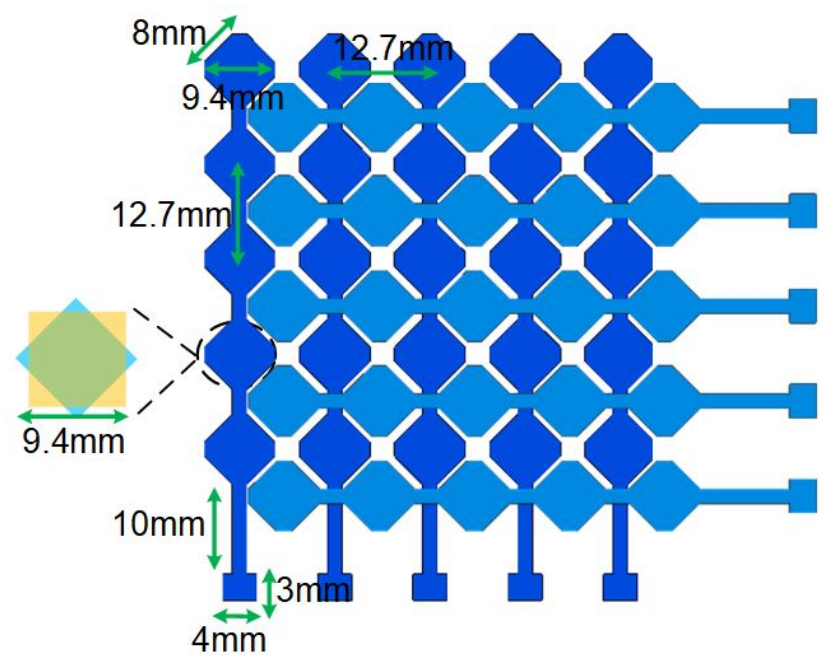

Figure S4. Specific dimensions of the electrode configuration. 